\title{
Study of Liver Function Tests in Patients with Long Standing Type 2 Diabetes Mellitus in Comparison to Healthy Individuals
}

\author{
Rohan Guptaํㅗㄱ, Supriya Prathibha Shankaranarayana Bhat ${ }^{2}$, Pratheeksha Rai Gangadhar ${ }^{3}$, \\ Ganaraj Kulamarva ${ }^{4}$, Adithi Kellari ${ }^{5}$, Prakash P.S. ${ }^{6}$ \\ 1, 2,3, 5, 6 Department of General Medicine and Gastroenterology, K.S. Hegde Medical Academy \\ and Charitable Hospital, Nitte (Deemed to Be University), Deralakatte, Mangalore, Karnataka, \\ India. ${ }^{4}$ Department of Medical Gastroenterology, K.S. Hegde Medical Academy and Charitable \\ Hospital, Nitte (Deemed to Be University), Deralakatte, Mangalore, Karnataka, India.
}

\section{ABSTRACT}

\section{BACKGROUND}

Type 2 diabetes mellitus is fast emerging as a cause of non-alcoholic fatty liver disease (NAFLD) in the world. This study was undertaken to study the relationship between the derangement of liver function and long standing diabetes mellitus, to evaluate as to whether liver function tests should be included as a part of routine investigations in diabetic patients and study the relation, if any, between long standing diabetes mellitus and presence of fatty liver.

\section{METHODS}

This study is a hospital based observational case control study conducted over a period of one and a half years between January 2018 and June 2019. 100 patients with long standing type 2 diabetes mellitus from the in-patients were included as tests and 100 healthy patients from the out-patient department were recruited as controls after fulfilling the inclusion and exclusion criteria.

\section{RESULTS}

Majority of the patients in the study in both cases and controls belonged to the middle aged group. $25 \%$ of the cases belonged to the age group of 41 to 50 years with $28 \%$ of the controls belonging to the same age group. $28 \%$ of patients in the case group and $34 \%$ of patients in the control group were aged between 51 and 60 years. $50 \%$ of the diabetic population was found to have NAFLD in comparison to $23 \%$ healthy population. $16(76.2 \%)$ diabetic patients with a deranged serum glutamicoxaloacetic transaminase (SGOT) and $9(64.3 \%)$ patients with a deranged Serum glutamic pyruvic transaminase (SGPT) were found to have a fatty liver on ultrasonography which was statistically significant. Furthermore, alkaline phosphatase (ALP) was found to be deranged in $10(10 \%)$ diabetic patients but none of the healthy patients.

\section{CONCLUSIONS}

NAFLD is widely present in patients with long standing type 2 diabetes mellitus. We observed in this study that it is the duration of diabetes mellitus which associates with the presence of fatty liver. Further, since NAFLD carries the risk of turning malignant in cases of steatohepatitis, it is suggested that liver function tests and ultrasonography should be made a part of the examination protocol in all diabetic patients.

\section{KEY WORDS}

Liver Function Tests, Type 2 Diabetes Mellitus, Fatty Liver, Ultrasonography, NAFLD
Corresponding Author:

Dr. Ganaraj Kulamarva, Assistant Professor,

Department of Medical Gastroenterology, K.S. Hegde Medical Academy and Charitable Hospital, Nitte (Deemed to Be University), Deralakatte, Mangalore, Karnataka, India.

E-mail: drgansy01@yahoo.co.in

DOI: 10.14260/jemds/2021/64

How to Cite This Article:

Gupta R, Bhat SPS, Gangadhar PR, et al. Study of liver function tests in patients with long standing type 2 diabetes mellitus in comparison to healthy individuals. J Evolution Med Dent Sci 2021;10(05):289293, DOI: 10.14260/jemds/2021/64

Submission 30-09-2020,

Peer Review 01-12-2020,

Acceptance 07-12-2020,

Published 01-02-2021.

Copyright (C) 2021 Rohan Gupta et al. This is an open access article distributed under Creative Commons Attribution License [Attribution 4.0 International (CC BY 4.0)] 


\section{BACKGROUND}

Diabetes mellitus is a common, chronic disorder resulting in hyperglycaemia due to either a drop in production of insulin due to destruction of insulin secreting beta cells of pancreas or due to decreased sensitivity to insulin in the body. ${ }^{1}$ Type 2 diabetes mellitus is caused by the body's ineffective use of insulin. It usually results from excess weight and physical inactivity. ${ }^{2}$ Type 2 diabetes mellitus is more common of the two and commonly prevalent amongst adults, the other one being type 1 diabetes mellitus. Currently, the accepted diagnostic criteria for diabetes mellitus consists of one of the following: 1) a random blood sugar greater than $200 \mathrm{mg} / \mathrm{dL}$ in a symptomatic patient or 2) HbA1c greater than $6.5 \%$ or 3 ) a fasting plasma glucose greater than $126 \mathrm{mg} / \mathrm{dL}$ or 4) in a patient who has symptoms of hyperglycaemia, a random plasma glucose $\geq 200 \mathrm{mg} / \mathrm{dL}^{3}$ The number of people with diabetes has risen from 108 million in 1980 to 463 million in 2019. This has increased drastically in over the past 3 decades, more so in middle and lower-income countries. ${ }^{4}$ Long standing diabetes mellitus is often associated with multiple microvascular as well as macrovascular complications. Amongst these, diabetic retinopathy is the most commonly associated long-term complication with an estimated $35 \%$ of diabetics developing proliferative or non-proliferative retinopathy over longer durations of time. ${ }^{5}$ It is also associated with a higher risk of cardiovascular diseases compared to adults without diabetes mellitus. ${ }^{6}$

Non-alcoholic fatty liver disease (NAFLD) is the most common chronic liver condition of adults in developed countries. ${ }^{7}$ It is defined as a clinical syndrome characterized by predominant macrovesicular steatosis of the liver. ${ }^{8}$ According to the guidelines being followed currently, the diagnosis of NAFLD is based on the following criteria: 1) the presence of hepatic steatosis (> $5 \%$ of hepatocytes determined by histology or $>5.6 \%$ determined by imaging techniques),2) no significant alcohol consumption (not more than 21 drinks / week for men and 14 drinks / week for women), and 3) no other possible aetiologies for hepatic steatosis.

Since the exact aetiology of NAFLD in the setting of diabetes mellitus is poorly understood, till recently it was not viewed to have significant association with type 2 diabetes mellitus. But in the past few years, it has been observed in multiple studies that patients who have long standing diabetes mellitus are more prone to develop more severe forms of NAFLD compared to a healthy person with no comorbidities. ${ }^{8}$ Although NAFLD has over the time been widely considered as a benign entity, its association with type 2 diabetes mellitus has been coming to the fore in recent times. It is now being accepted that patients with type 2 diabetes mellitus and associated NAFLD are at a higher risk of cardiovascular complications and chronic liver diseases which may further progress to cirrhosis and finally towards hepatocellular carcinoma. ${ }^{2}$ This brings to light the massive burden of association of type 2 diabetes mellitus with NAFLD. The number of studies from the Asian subcontinent and in particular South India are limited at the moment. This study has been taken up to determine if there is a correlation between long standing type 2 diabetes mellitus and derangement of liver function test (LFT) in the Indian population.

\section{METHODS}

This is a hospital based, observational case control study During the time period of January 2018 to June 2019, the patients with long standing ${ }^{1}$ type 2 diabetes mellitus, for more than 5 years for the purpose of our study who were admitted in Justice K.S. Hegde charitable hospital, Mangalore were questioned, evaluated and examined. The study was approved by ethics committee and informed consent was obtained. Simple random sampling was done and after excluding patients with liver diseases, diabetes mellitus for less than 5 year duration, chronic alcoholics and patients on drugs known to cause hepatotoxicity, the rest of the patients were included in the test group. These patients underwent liver function tests and ultrasonogram (USG) of the abdomen. During the same duration, healthy individuals with no underlying comorbidities, diabetes mellitus, liver disease or history of regular alcohol intake who visited the OPD in Justice K.S. Hegde charitable hospital, Mangalore were included in the control group after taking informed consent and underwent liver function tests and USG abdomen, same as the test group. A total of 100 patients were included in the study group and 100 were qualified for the control group as well.

\section{Sample Size}

Based on the anticipated proportion of $15 \%$, precision of $5 \%$ and level of significance of $5 \%$, a sample size of 196 was arrived at while keeping in consideration the turnover of in patients with long standing diabetes mellitus and was rounded off to 200 . Thus, a total of 200 patients with 100 test subjects and 100 controls will be included in the study. The formula to estimate the sample size is as follows -

$\mathrm{n}=\frac{\left[\mathrm{z}^{2} 1-\alpha / 2 P(1-\mathrm{P})\right]}{\mathrm{d}^{2}}$

For estimation of single proportion where,

$\mathrm{Z}=$ Standard normal variant

$\alpha=$ Level of significance (5\%)

$\mathrm{d}=$ Precision $(5 \%)$

$\mathrm{P}=$ Anticipated proportion $(15 \%)$

$\mathrm{n}=(1.96)^{2} \times 0.15 \times(1-0.15) /(0.05)^{2}=196$

\section{Inclusion Criteria}

1. Patients above 18 years of age with diabetes mellitus type 2 for more than 5 years constituted the test group.

2. Healthy individuals above 18 years of age, without any comorbidities visiting for routine health check-up constituted the control group.

\section{Exclusion Criteria}

1. Patients with type 1 diabetes mellitus

2. Patients with type 2 diabetes mellitus of less than 5 years

3. Patients with liver diseases 
4. Patients with renal diseases

5. Chronic alcoholics

6. Non-consenting patients

\section{Method of Data Collection}

During the duration of the study, the patients with long standing ${ }^{1}$ type 2 diabetes mellitus who were admitted in the hospital were questioned, evaluated and examined. Upon satisfying the inclusion criteria, an informed consent was taken and they were subjected to interview for history of the disease, clinical examination and a blood sample was taken in a plain vial after which it was analysed in the biochemistry lab in Roche Hitachi Cobas chemsitry analyser c-311 for liver function tests. Additionally, they were also subjected to ultrasonography of the liver by the radiologists to look for any alteration of echotexture of liver or for evidence of presence of fatty liver

\section{Statistical Analysis}

The results were tabulated and further analysed in International Business Machines Statistical Package for the Social Sciences (IBM SPSS) version 24 with chi square test and $P$ value of $<0.05$ being considered significant.

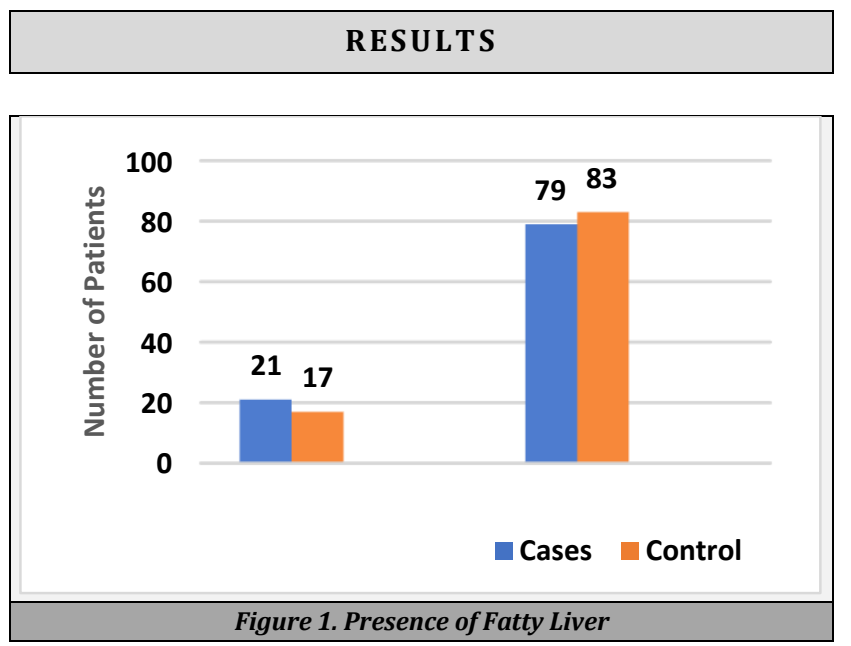

\begin{tabular}{|ccccccc|}
\hline ALP Deranged & \multicolumn{2}{c}{ Cases } & \multicolumn{2}{c}{ Control } & P-Value \\
& $\mathbf{N}=\mathbf{1 0 0}$ & $\mathbf{\%}$ & $\mathbf{N}=\mathbf{1 0 0}$ & $\mathbf{\%}$ & \\
\hline Yes & 10 & $10 \%$ & 0 & $0 \%$ & 0.0001 \\
No & 90 & $90 \%$ & 100 & $100 \%$ & \\
\hline Total & $\mathbf{1 0 0}$ & $\mathbf{1 0 0} \%$ & $\mathbf{1 0 0}$ & $\mathbf{1 0 0} \%$ & \\
\hline Table 1. Derangement of Alkaline Phosphatase (ALP) &
\end{tabular}

\begin{tabular}{|ccccccc|}
\hline Case Group & \multicolumn{5}{c}{ Ultrasonography } & P-Value \\
& & Fatty & \multicolumn{4}{c|}{ Normal } \\
SGOT Deranged & Yes & 16 & $76.2 \%$ & 5 & $23.8 \%$ & .007 \\
& No & 34 & $43.0 \%$ & 45 & $57.0 \%$ & \\
SGPT Deranged & Yes & 9 & $64.3 \%$ & 5 & $35.7 \%$ & 0.249 \\
& No & 41 & $47.7 \%$ & 45 & $52.3 \%$ & \\
ALP Deranged & Yes & 6 & $60.0 \%$ & 4 & $40.0 \%$ & 0.505 \\
& No & 44 & $48.9 \%$ & 46 & $51.1 \%$ & \\
\hline Table 2. Statistical Significance of Deranged Liver Function Test \\
(LFT) in Correlation to Presence of Fatty Liver in Cases \\
\hline
\end{tabular}

In comparison to the control population which has only 23 $\%$ patients with fatty liver on screening via ultrasonography, $50 \%$ of the patients with long standing diabetes mellitus had
NAFLD. This finding was statistically significant with a $P$ value of 0.0001 .

ALP derangement was found in $10 \%$ of the test population whereas none of the patients in the control group had derangement of ALP. This is statistically significant and suggests that ALP is deranged in patients with long standing type 2 diabetes mellitus.

In the case group, out of 21 patients who had a deranged SGOT, $16(76.2 \%)$ were found to have the presence of fatty liver as well. Using chi square / Fishers exact test, the P value was determined to be 0.007 and of high significance. Even though, 9 patients with SGPT derangement and 6 with ALP derangement were found to have fatty liver, these were not considered statistically significant. By this we infer that diabetic patients with high SGOT have an increased chance of presenting with fatty liver in comparison to patients with normal SGOT. The same conclusion cannot be drawn for SGPT and ALP.

\begin{tabular}{|c|c|c|c|c|c|c|}
\hline \multicolumn{2}{|c|}{ Control Group } & \multicolumn{4}{|c|}{ Ultrasonography } & \multirow{3}{*}{ Value } \\
\hline & & \multicolumn{2}{|c|}{ Fatty } & \multicolumn{2}{|c|}{ Normal } & \\
\hline & & $\mathrm{N}=100$ & $\%$ & $\mathrm{~N}=100$ & $\%$ & \\
\hline \multirow[t]{2}{*}{ SGOT Deranged } & Yes & 11 & $64.7 \%$ & 6 & $35.3 \%$ & 0.001 \\
\hline & No & 12 & $14.5 \%$ & 71 & $85.5 \%$ & \\
\hline \multirow[t]{2}{*}{ SGPT Deranged } & Yes & 13 & $59.1 \%$ & 9 & $40.9 \%$ & 0.001 \\
\hline & No & 10 & $12.8 \%$ & 68 & $87.2 \%$ & \\
\hline \multirow[t]{2}{*}{ ALP Deranged } & Yes & 0 & $0 \%$ & 0 & $0 \%$ & \\
\hline & No & 23 & $23.0 \%$ & 77 & $77.0 \%$ & 0.001 \\
\hline
\end{tabular}

It was observed that in the control group, 11 (64.7\%) patients with a deranged SGOT had fatty liver and 13 (59.1\%) patients with a deranged SGOT were found to have a fatty liver on ultrasonography. These derangements in LFTs were found to be statistically significant when looking for fatty liver. From this, it can be inferred that apparently healthy individuals who have deranged SGOT and SGPT values have a higher chance of having an underlying fatty liver in comparison to individuals with normal liver enzymes, hence, these patients need to be evaluated further with ultrasonography.

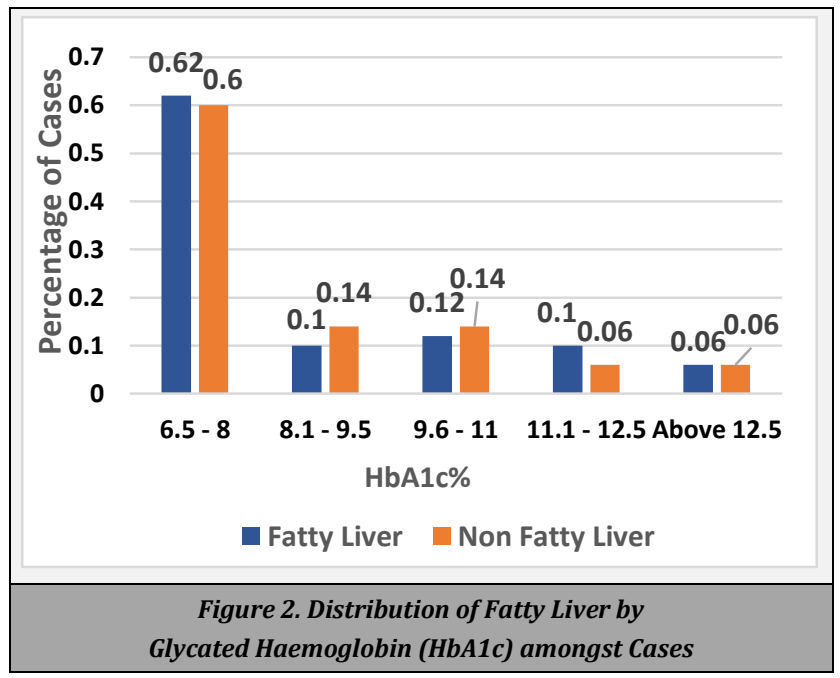

We found that the distribution of patients with type 2 diabetes with fatty liver was similar in patients without fatty liver, across various degrees of glycaemic control. 


\section{DISCUSSION}

In this study, 100 patients with long standing type 2 diabetes mellitus underwent liver function tests along with ultrasonography of the liver. In parallel, 100 control subjects were recruited from the clinics who underwent similar investigations.

Being deficit in data across our country, we were aiming to evaluate if there was a significant role of type 2 diabetes mellitus in precipitating NALFD. When we analysed derangement of liver enzymes in our case group, we found that SGOT and SGPT were deranged in $21 \%$ and $14 \%$ patients respectively. When we analysed derangement of liver enzymes in our case group, we found that SGOT and SGPT were deranged in $21 \%$ and $14 \%$ patients respectively but, this was not statistically significant when compared to the control group in which $17 \%$ and $22 \%$ patients had deranged SGOT and SGPT respectively. These findings were similar to a study done in Myanmar by Han Ni et al.10 (2012) which concluded that SGOT was deranged in $14.8 \%$ patients with long standing type 2 diabetes mellitus and SGPT was deranged in $18.5 \%$ of the diabetics.

We also found that the derangement of ALP was significant in our study with ALP being raised in $10 \%$ of the patients in the case group where as we did not find any derangement in our control groups from which we could infer that liver dysfunction is more likely in diabetic population compared to the healthy population. This finding though has been substantiated in another study by Sean Chun-Chang Sen et al ${ }^{11}$ in which 6,555 diabetic patients were included and $7 \%$ were found to have a derangement of ALP.

Furthermore, in our study which carried out ultrasonography of the diabetic patients, we found that $50 \%$ of them had fatty liver. In a study titled "Type 2 diabetes mellitus and non-alcoholic fatty liver disease: a systematic review and meta-analysis" by Nasrin Amiri et al. ${ }^{12}$ 10,897 patients were included in the meta-analysis. This was aimed to assess if there was any significant co-relation between the presence of type 2 diabetes mellitus and NAFLD when compared to other types of diabetes. The pooled prevalence of fatty liver in the studied population was found to be $54 \%$ and they concluded that the prevalence of NAFLD in patients with type 2 diabetes mellitus was significantly higher than other types.

Loomba ${ }^{8}$ also concluded that the presence of fatty liver depends on the duration of diabetes mellitus rather than the glycaemic control of the patients, the findings of which are similar to our study.

Even though there was derangement of liver function tests in few of these patients, yet we found that in spite of having a normal liver function the patients could still have NAFLD which would have been missed out and had the screening method of only enzymatic derangement been applied. Liver ultrasonography is an effective method in screening. $76.2 \%$ patients in the test group and $64 \%$ individuals in control group had fatty liver in the presence of deranged SGOT. Similarly, $64.3 \%$ patients in test group and $59.1 \%$ individuals in control group had fatty liver in the presence of deranged SGPT. From this, it can be inferred that irrespective of presence of diabetes mellitus or not, individuals who have deranged SGOT and SGPT values have a higher chance of having an underlying fatty liver in comparison to individuals with normal liver enzymes, hence, these patients need to be evaluated further with ultrasonography.

\section{CONCLUSIONS}

It can be inferred from the study that there is a significant association between patients with long standing type 2 diabetes mellitus and non-alcoholic fatty liver disease. We observed in this study that the glycaemic control or glycated haemoglobin levels is not related to the presence of fatty liver but rather, it is the duration of diabetes mellitus which is associated with the presence of fatty liver. Considering the significant association between NAFLD and type 2 diabetes mellitus, it is warranted that studies at a larger scale should be done in Indian population where the data is deficient. Screening method of enzyme derangement is not accurate as more than half the patients in our study had NAFLD in spite of normal liver enzymes. It should also become a regular practice to screen patients with type 2 diabetes mellitus for NAFLD by ultrasonogram to improve the overall outcome of the disease. Further, since NAFLD carries the risk of turning malignant in cases of steatohepatitis, it needs to be looked for in all diabetic patients.

Data sharing statement provided by the authors is available with the full text of this article at jemds.com.

Financial or other competing interests: None.

Disclosure forms provided by the authors are available with the full text of this article at jemds.com.

\section{REFERENCES}

[1] Song S, Wang B, Zhang X, et al. Long-term diabetes mellitus is associated with an increased risk of Pancreatic cancer: a meta-analysis. PloS One 2015;10(7):e0134321.

[2] Harrington FR, Wolfenden H, Makaya T. Type 2 diabetes presenting with hyperglycaemic hyperosmolar state in an adolescent renal transplant patient. BMJ Case Rep 2015;2015:bcr2014207124.

[3] Jameson JL. Harrison's principles of internal medicine. 20th edn. New York: McGraw - Hill Education 2018: p. 2.

[4] Roglic G. Global report on diabetes. Geneva, Switzerland: World Health Organization 2016: p. 86.

[5] Yau JWY, Rogers SL, Kawasaki R, et al. Global prevalence and major risk factors of diabetic retinopathy. Diabetes Care 2012;35(3):556-64.

[6] Sarwar N, Gao P, Seshasai SRK, et al. Diabetes mellitus, fasting blood glucose concentration, and risk of vascular disease: a collaborative meta - analysis of 102 prospective studies. Lancet 2010;375(9733):2215-22.

[7] Bril F, Cusi K. Management of nonalcoholic fatty liver disease in patients with type 2 diabetes: a call to action. Diabetes Care 2017;40(3):419-30.

[8] Loomba R, Sanyal AJ. The global NAFLD epidemic. Nat Rev Gastroenterol Hepatol 2013;10(11):686-90.

[9] Trombetta M, Spiazzi G, Zoppini G, et al. Review article: type 2 diabetes and chronic liver disease in the Verona 
diabetes study. Aliment Pharmacol Ther 2005;22 Suppl 2):24-7.

[10] Ni H, Soe HHK, Htet A, et al. Determinants of abnormal liver function tests in diabetes patients in Myanmar. Int J Diabetes Res 2012;1(3):36-41.

[11] Chen SCC, Tsai SP, Jhao J Y, et al. Liver fat, hepatic enzymes, alkaline phosphatase and the risk of incident type 2 diabetes: a prospective study of 132,377 adults. Sci Rep 2017;7(1):4649.

[12] Atan NAD, Koushki M, Motedayen M, et al. Type 2 diabetes mellitus and non-alcoholic fatty liver disease: a systematic review and meta - analysis. Gastroenterol Hepatol Bed Bench 2017;10(Suppl 1):S1-7. 